\title{
Comparison of systemic and mucosal vaccination: impact on intravenous and rectal SIV challenge
}

\author{
DL Bolton ${ }^{1}$, K Song ${ }^{1}$, RL Wilson ${ }^{2}$, PA Kozlowski ${ }^{2}$, GD Tomaras ${ }^{3}$, BF Keele ${ }^{4}$, RV Lovingood ${ }^{3}$, S Rao $^{5}$ \\ and $M$ Roederer ${ }^{1}$
}

Mucosal tissues are the primary route of transmission for most respiratory and sexually transmitted diseases, including human immunodeficiency virus. We aimed to generate strong mucosal immune responses to simian immunodeficiency virus (SIV) in rhesus macaques by targeting recombinant adenovirus serotype 5 (rAd5) to the lung. The immunogenicity and efficacy of aerosol (AE) vaccination was compared with intramuscular (IM) delivery in either an intravenous (IV) or

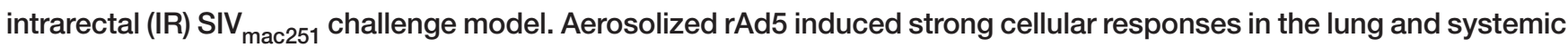
humoral responses equivalent to IM. Strikingly, all immunization groups controlled acute viremia in the IV challenge model by 1-2 logs. By contrast, after IR challenge, only peak viremia was reduced by immunization, with no significant effect on SIV infection acquisition rate or mucosal CD4 ${ }^{+}$T-cell preservation. Improved disease outcome was associated with pre-challenge cellular and humoral responses, while post-challenge T-cell responses were highly correlated with viremia control. The similar outcomes achieved by systemic and airway mucosal immunization support AE delivery as a safe, effective, and less invasive alternative to parenteral vaccination.

\section{INTRODUCTION}

A highly effective vaccine that generates broadly reactive responses and confers sterilizing immunity against human or simian immunodeficiency virus (HIV or SIV) has yet to be developed. It is widely believed that immune responses must function at the mucosal barrier to disrupt sexual transmission. This is supported by the unique ability of vaccine platforms eliciting either differentiated memory mucosal T cells or rectal antibodies to diminish rectal SIV transmission in non-human primates. ${ }^{1-3}$ Similarly, partial protection against vaginal SIV transmission has only been achieved with vector delivered mucosally by intranasal administration. ${ }^{4,5}$ By targeting mucosal surfaces, these vaccines presumably focus the immune response to the site of pathogen entry, thereby curbing early virus replication.

Replication-deficient recombinant adenovirus (rAd) is a potent vaccine platform for inducing cellular immune responses and has been the focus of many experimental vaccine studies in non-human primate and human clinical trials. With the failure of an rAd serotype 5 (rAd5) vector to protect against HIV acquisition in humans and the potential confounding issue of Ad5 seroprevalence, ${ }^{6}$ this vector will likely not advance beyond clinical trials. However, vectors derived from less prevalent adenovirus serotypes, such as $\operatorname{Ad} 26, \operatorname{Ad} 28, \operatorname{Ad} 35$, simian Ad, and chimpanzee Ad, are also immunogenic, with some showing control of SIV. ${ }^{7,8}$

Assessment of rAd vector-mediated immunogenicity and protection from challenge is typically based on intramuscular (IM) administration, which generates strong systemic T-cell responses but modest mucosal responses. We hypothesized that mucosal delivery of rAd5 would enhance mucosal immunity and control HIV/SIV replication to a greater extent than IM delivery. Previously, replication-competent rAd encoding SIV proteins targeting multiple mucosal sites, including oral, nasal, and tracheal tissue, in combination with an Env protein boost, was able to reduce viral replication and block transmission after

\footnotetext{
${ }^{1}$ ImmunoTechnology Section, Vaccine Research Center, NIAID, NIH, Bethesda, Maryland, USA. 2Department of Microbiology, Immunology, and Parasitology, Louisiana State University Health Sciences Center, New Orleans, Louisiana, USA. ${ }^{3}$ Duke Human Vaccine Institute, Duke University, Durham, North Carolina, USA. ${ }^{4}$ AIDS and Cancer Virus Program, SAIC Frederick Inc., NCI-Frederick, Frederick, Maryland, USA. 5 Laboratory Animal Medicine, Vaccine Research Center, Bethesda, Maryland, USA. Correspondence: M Roederer (Roederer@nih.gov) 
rectal SIV $_{\text {mac251 }}$ challenge. ${ }^{9}$ However, replication-competent rAd has not been developed as extensively as non-replicating Ad vectors and may face safety concerns in humans. Further, while oral delivery of rAd5 may protect against oral $\mathrm{SIV}_{\text {mac239 }}$ challenge, ${ }^{10}$ a more physiological challenge model is warranted. Mucosal rAd immunization has yet to be tested for protection against a mucosal rectal or vaginal SIV challenge, the best currently available models of HIV transmission.

We recently characterized the immunogenicity of aerosolized rAd5 in rhesus macaques and demonstrated complete vaccinemediated protection against influenza challenge in ferrets. ${ }^{11}$ Here we present efficacy data comparing aerosol (AE) and IM delivery of rAd5 in SIV mac251 $_{1}$ intravenous (IV) and limitingdose intrarectal (IR) challenge models. The rationale for AE delivery as a mucosal route is compelling. Targeting airway mucosal sites generates distal humoral responses at vaginal and rectal mucosa and more robust mucosal T-cell responses than IM delivery. ${ }^{2,11-13}$ Moreover, it has been successfully used for measles immunization in children without adverse effects. ${ }^{14}$ In the IV challenge setting, we also compared each route with and without a systemic DNA prime to augment $\mathrm{CD} 4{ }^{+}$T-helper and humoral responses. Both AE and IM rAd5 achieved similar levels of protection against either SIV challenge route, reducing peak plasma viremia and mucosal $\mathrm{CD} 4^{+} \mathrm{T}$-cell depletion to the same extent. Strikingly, blood cellular responses at the time of challenge, which were undetectable in the AE group, did not predict virus control. These data indicate that $\mathrm{AE}$ vaccine vectors are potentially useful immunization platforms against SIV/HIV.

\section{RESULTS}

\section{Experimental schema}

To determine how mucosal and systemic immunizations compare in IV and IR challenge models, we immunized rhesus macaques by $\mathrm{AE}$ or IM with $5 \times 10^{10} \mathrm{PU}$ each of rAd5 encoding SIV gag-pol and env or empty rAd5 twice 3-4 weeks apart (Figure 1a). Two groups also received a DNA prime consisting of plasmids encoding SIV $_{\text {mac239 }}$ gag-pol-nef and env 6 months prior to rAd5 in the IV challenge study, as a systemic immunization may prime mucosal rAd5. The IR SIV $\operatorname{mac} 251_{\text {challenge }}$ was administered by repetitive delivery of a dose previously determined to achieve $50 \%$ infection after each application. The vaccine platforms used in the IV and IR infection studies were identical with the following exceptions: (i) there was no DNA prime in the IR study, as this had a minimal effect on plasma viremia in the IV system; and (ii) the two sham groups in the IR study received empty rAd5 by either AE or IM. For most analyses, including viremia, these two sham subgroups were combined when no difference was observed between them.

\section{Humoral responses following immunization}

Antibody responses correlate with protection in macaque SIV challenge models. ${ }^{3,9,15,16}$ We measured SIV Env- and GagPolspecific immunoglobin $\mathrm{G}(\mathrm{IgG})$ and immunoglobin A (IgA) by enzyme-linked immunosorbent assay before and after rAd5 vaccination (Figure 2). Env-specific serum IgG responses to

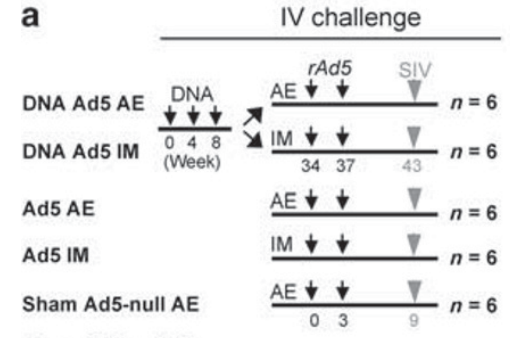

b Repeated IR challenge

Sham Ad5-null IM

Figure 1 Experimental schema for the $\mathrm{SIV}_{\text {mac251 }}$ challenge studies. (a) In the intravenous (IV) study, animals were immunized (small black arrows) with the indicated vaccine component (DNA or rAd5) at the indicated week. SIV challenge (gray arrows) was administered 6 or 7.5 weeks after the second rAd5 immunization for the IV and limiting repeated intrarectal (IR) challenges (b), respectively. Repeated IR challenges were administered as described in the Methods. Vaccination group names are indicated on the left. AE, aerosol; IM, intramuscular.

unprimed rAd5 were similar in IM and AE groups, ranging from 1 to $10 \mu \mathrm{g} \mathrm{mg}^{-1}$ of total Ig (Figure 2a). Moreover, DNA primed responses to rAd5 similarly, regardless of rAd5 delivery route $\left(10-100 \mu \mathrm{g} \mathrm{mg}^{-1}\right)$, and these responses were significantly greater than those in unprimed animals ( 5 weeks post-rAd: IM, $P=0.03$; AE, $P=0.0006)$. Although less immunogenic than Env, the same trend was observed for IgG responses to GagPol (Figure 2b). Serum IgA to both GagPol and Env also did not differ with rAd5 delivery route (Figure 2c). However, while DNA priming did not significantly improve Env-specific IgA in the serum, rectal mucosal Env-specific IgA was elevated in the DNA-primed groups compared with unprimed animals $(P=0.04$, Wilcoxon; Figure 2d), as was serum IgA to GagPol (Figure 2c, $P=0.04$ ). Despite these robust responses, no serum-neutralizing antibody

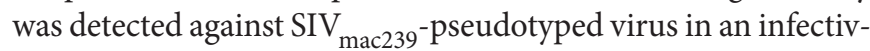
ity reduction assay (data not shown).

\section{Cellular responses following immunization}

SIV-specific $\mathrm{CD} 4^{+}$and $\mathrm{CD} 8^{+} \mathrm{T}$ cells were measured in cryopreserved peripheral blood mononuclear cells by in vitro antigen stimulation with Gag and Env peptide pools, followed by intracellular cytokine staining for interferon- $\gamma$, interleukin- 2 , and tumor necrosis factor- $\alpha$. To assess the total magnitude of cellular immune responses, we enumerated all cytokine-producing cells responding to either Gag or Env peptide pools. IM immunization elicited greater $\mathrm{CD} 8^{+} \mathrm{T}$-cell responses than $\mathrm{AE}$ delivery (up to $2.6 \%$ of circulating $\mathrm{CD} 8{ }^{+} \mathrm{T}$ cells at 5 weeks post-rAd5 prime), while $\mathrm{CD}^{+}$responses were not significantly different between the groups (Figure 3a and $\mathbf{b}$ ). Both IM and AE rAd boosted DNA-primed T-cell responses; however, in unprimed animals, IM but not AE generated stable circulating T-cell responses, which were detectable 9 weeks after the rAd5 prime (the day of SIV challenge).

Mucosal T-cell responses were similarly measured in bronchoalveolar lavage (BAL) single-cell suspensions from three animals per vaccine group. Consistent with previous results, ${ }^{11} \mathrm{AE}$ delivery of rAd5 induced a very high frequency of SIV-specific cells (up to 25 and $50 \%$ of $\mathrm{CD}^{+}$and $\mathrm{CD} 8^{+} \mathrm{T}$ cells, respectively) 

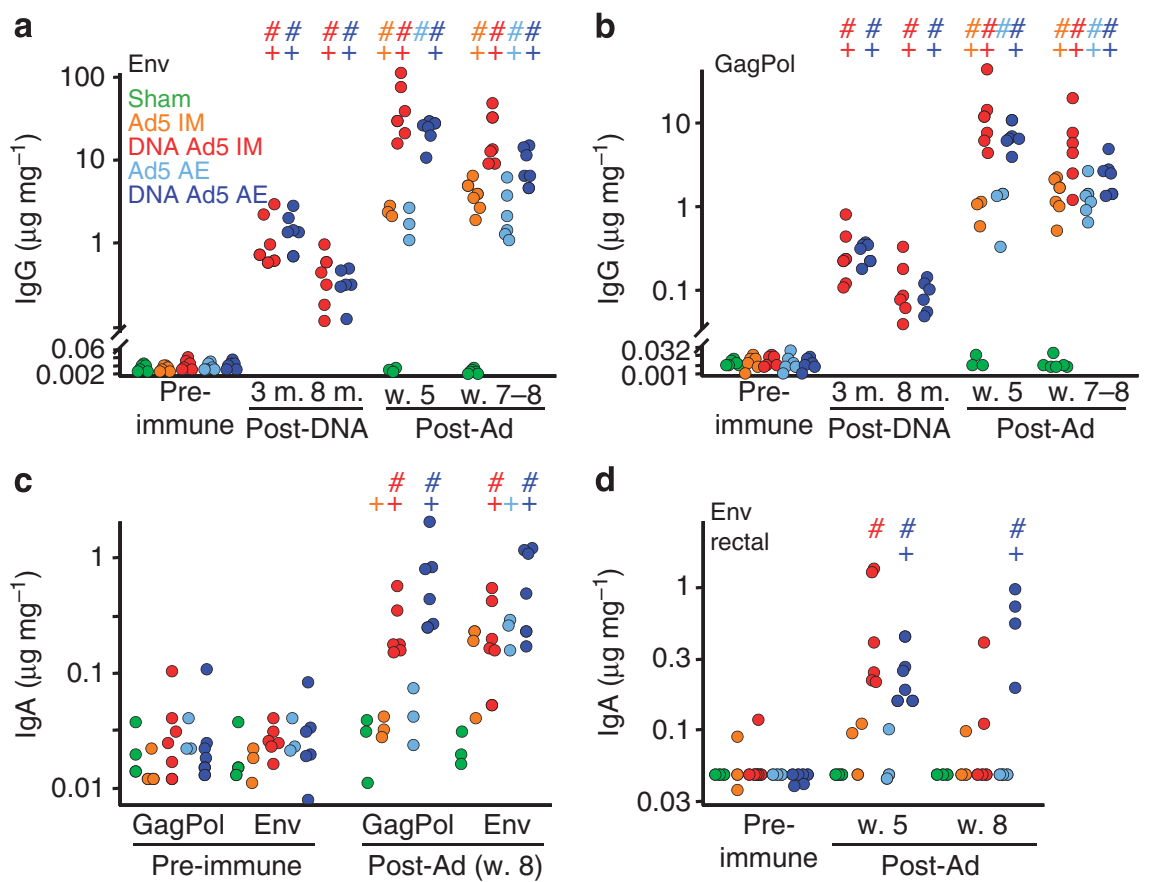

Figure 2 Humoral responses to simian immunodeficiency virus (SIV) following aerosol (AE) and intramuscular (IM) recombinant adenovirus serotype 5 (rAd5) immunization with and without DNA priming. (a) Serum Env-specific and GagPol-specific (b) immunoglobin G (IgG) in each vaccination group plotted after the indicated immunization time point as $\mu \mathrm{g}$ specific antibody per mg of total lgG (M., month; W., week; relative to first DNA or rAd5 immunization). Animal groups are coded by color as indicated by the legend text. Only three animals were sampled for the Ad5 alone groups at week 5 post-Ad. (c) Env- and GagPol-specific serum IgA pre-immunization and at week 8 post-Ad. IgA responses were only measured for three animals from the Ad5 alone groups. (d) Rectal Env-specific IgA at 5 and 8 weeks after $r A d$. Only rectal secretions $>0.145 \mu \mathrm{g}$ Env-specific IgA per $\mathrm{mg}$ total IgA contained significant antibody. $(+)$ and $(\#)$ indicate significant difference $(P<0.05)$ by Student's $t$-test and Wilcoxon rank sum, respectively, from the pre-immune time point. Animals for which insufficient total IgA was detected ( $n=1$, DNA Ad5 IM, $n=2$, DNA Ad5AE) at week 8 post-Ad are omitted.

8 weeks after the first rAd5 immunization (Figure 3c). These responses were similar in DNA-primed and unprimed animals. By contrast, IM delivery induced a much smaller response (0$1 \%$ of $\mathrm{CD}^{+}$and $2-5 \%$ of $\mathrm{CD}^{+}{ }^{+} \mathrm{T}$ cells) in unprimed animals and only slightly greater $\mathrm{CD} 8{ }^{+}$responses when primed with DNA $(P=0.06)$. Unsurprisingly, AE delivery of the vaccine was more effective at inducing lung mucosal T-cell responses than IM delivery, although DNA-primed IM rAd5 achieved a similar proportion of antigen-specific $\mathrm{CD} 8^{+} \mathrm{T}$ cells. Despite these robust lung responses, only the DNA rAd5 IM group mounted substantial CD8 ${ }^{+}$T-cell responses in the jejunum compared with the unimmunized animals (Supplementary Figure S1 online), confirming that the respiratory and gastrointestinal mucosa cellular responses are distinct. ${ }^{17}$

The extent to which the antigen-specific $\mathrm{T}$ cells produce multiple cytokines correlates with disease protection in several animal models and human infections. ${ }^{18,19}$ The SIV-specific $\mathrm{T}$ cells elicited by our vaccine regimens in the BAL were highly polyfunctional-at least $20-25 \%$ of the $\mathrm{CD} 4^{+}$and $\mathrm{CD} 8^{+}$subsets simultaneously expressed interferon- $\gamma$, interleukin-2, and tumor necrosis factor- $\alpha$ (Figure 3d). Although the cytokine expression patterns were not significantly different between immunization groups, our data indicate that DNA priming may augment the protective triple and interferon- $\gamma^{+}$double-positive $\mathrm{CD} 4^{+}$ populations.

We also considered whether SIV-specific responses to each immunogen varied across the vaccine regimens. The proportion of the BAL response mounted to Gag, Pol, or Env was not significantly different between the groups 5 weeks after the rAd5 boost, with the majority of $\mathrm{CD} 4^{+}$and $\mathrm{CD} 8^{+} \mathrm{T}$ cells specific for Env (Figure 3e). Pol-specific $\mathrm{CD} 8^{+} \mathrm{T}$ cells trended toward greater prevalence in the AE rAd5 group, but this was not statistically significant. Similarly, the blood T-cell responses were heavily skewed toward Env, with no large difference in antigen targeting among the groups (data not shown).

\section{Post-challenge viremia}

To assess vaccine efficacy, macaques were challenged IV with $100 \mathrm{MID}_{50} \mathrm{SIV}_{\text {mac251 }}$ and plasma viremia measured by quantitative PCR for SIV gag RNA. Viremia was detected in all animals within 1 week of challenge (Figure 4a). As expected, ${ }^{20} \mathrm{IM}$ rAd5 immunization, with or without DNA prime, significantly reduced viremia by $1.5 \log$ relative to the peak observed for sham animals at day 14 . Surprisingly, AE rAd5 also lowered viremia by $1.0 \log$ at day 14 in DNA-primed and unprimed animals, despite the absence of SIV-specific T cells in the periphery at the time of challenge. DNA priming did not significantly impact acute viremia values, except by reducing day 10 (peak) viremia in the IM group by $0.8 \mathrm{log}$. Enhanced control of plasma viremia in the vaccine groups persisted throughout acute infection until about 6 months post-infection, at which point the sham group viral load declined to a similar set point as the vaccines (Figure $\mathbf{4 b}$ ). Major histocompatibility complex genotyping revealed five Mamu- $B^{\star} 29$ animals (from four of the vaccine groups) and one 

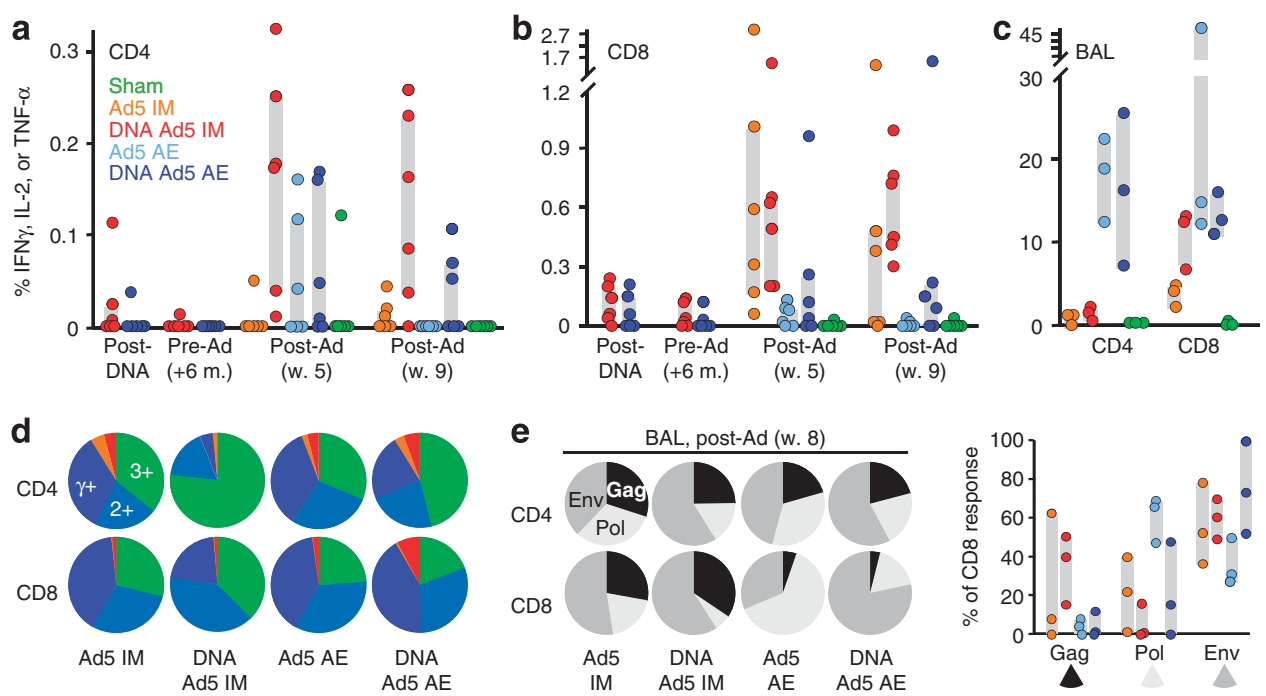

Figure 3 Simian immunodeficiency virus (SIV)-specific T-cell responses in peripheral blood and bronchoalveolar lavage (BAL) following recombinant adenovirus serotype 5 ( $r A d 5)$ vaccination. (a) $\mathrm{CD}^{+}$and $\mathrm{CD}^{+}$(b) T-cell responses in peripheral blood mononuclear cell isolated from each animal following immunization were measured in vitro by Gag and Env peptide pool stimulation and intracellular cytokine staining for interferon (IFN)- $\gamma$, tumor necrosis factor (TNF)- $\alpha$, and interleukin (IL)-2. The percentage of cells that produce one or more cytokines to either pool was summed as the total response. Gray bars indicate the interquartile range for each group. (c) BAL CD4 ${ }^{+}$and CD8 ${ }^{+}$SIV-specific T-cell responses measured as in a, with the inclusion of Pol-specific responses, 8 weeks after the first rAd5 immunization ( $n=3$ animals per group). (d) The cytokine profile of SIV-specific BAL T cells determined in $\mathbf{c}$ is shown as pie charts for $\mathrm{CD} 4^{+}$and $\mathrm{CD} 8^{+}$subsets. The fraction of responding cells that synthesize all three cytokines is depicted in green, IFN $\gamma$ and either IL-2 or TNF- $\alpha$ in light blue $(2+)$, and IFN $\gamma$ alone in dark blue. TNF- $\alpha^{+} I L-2^{+}$IFN $\gamma^{-}$populations are depicted in orange and single-positive TNF- $\alpha$ or IL-2 in red. (e) The average distribution of Gag-, Env-, and Pol-specific CD4 ${ }^{+}$and CD8 ${ }^{+}$T cells in BAL following rAd5 immunization. Bar graph depicts $\mathrm{CD} 8^{+}$targeting by animal for each vaccine group. AE, aerosol; IM, intramuscular; m., month; w., week.

Mamu- $B^{\star} 08$ animal (also from a vaccine group; Supplementary Figure S2A online). These alleles correlate with natural control of SIV replication in rhesus macaques; ${ }^{21}$ however, expression of these alleles was not associated with lower viremia (Supplementary Figure S2B online).

To compare viral replication among the vaccine groups following mucosal IR challenge, we first aligned viral load curves for each animal relative to peak viremia due to the variation in time to infection. When synchronized in this manner, peak plasma viral load was lower in both immunized groups relative to controls: 0.8 and $0.9 \log$ reductions by $\operatorname{AE}(P=0.026)$ and IM $(P=0.002)$, respectively (Figure $4 \mathbf{c}$ and $\mathbf{d})$. However, reduced viremia was not maintained at later points. Notably, peak viremia values were significantly lower following IR challenge than IV challenge, with the sham groups averaging $10^{7.2}$ and $10^{7.9}$ SIV copies per ml plasma, respectively $(P=0.008$; Figure 4e), suggesting different viral replication kinetics in these challenge models. Taking this into account, the magnitude of peak viremia reduction afforded by vaccination was similar between the studies: 1.2 and $0.9 \log$ reductions for the IV and IR challenges, respectively. Thus, rAd5-elicited immunity exerts the same level of virus control at peak viremia against systemic and mucosal SIV challenge.

There was no difference between the groups in the rate of IR SIV acquisition, with each SIV application infecting $~ 50 \%$ of animals in each group (Figure 4f). Moreover, there was no effect of vaccination on the number of SIV variants that established infection, based on single SIV genome sequencing of env in plasma at peak viremia (Figure 4g). Most animals were infected with one or a few variants and all groups averaged about two. Although these data suggest that vaccination did not limit early events in mucosal virus transmission, they indicate that the $\mathrm{SIV}_{\text {mac251 }}$ dosing scheme was a reasonable simulation of human sexual mucosal transmission with respect to the size of founder virus population.

\section{CD4 ${ }^{+} \mathrm{T}$-cell depletion}

Rapid destruction and elimination of memory $\mathrm{CD} 4^{+} \mathrm{T}$ cells, particularly in extra-lymphoid effector sites such as the small intestine and BAL, is a hallmark of pathogenic HIV and SIV infections. ${ }^{22-24}$ To assess the extent of CD4 ${ }^{+}$T-cell loss following IV and IR SIV challenge, we measured their proportion within the T-lymphocyte compartment of BAL and jejunum (Figure 5). One month after IV SIV challenge, $\mathrm{CD} 4{ }^{+} \mathrm{T}$ cells comprised $<1 \%$ of BAL T cells in the sham control group, and remained below $5 \%$ at time points up to 9 months post-infection (Figure 5a). However, before SIV challenge the mean percentage of $\mathrm{CD} 4^{+} \mathrm{T}$ cells in BAL of all $\mathrm{AE}$ animals dropped from $34(n=9)$ to $10(n=9)$, including the sham group (Figure 5a). Although consistent with preferential CD8 ${ }^{+} \mathrm{T}$-cell recruitment and accumulation in the lung caused by pulmonary rAd delivery, ${ }^{25}$ this confounds interpretation of the $\mathrm{CD} 4$ proportions. To account for this effect and quantify the SIV-induced CD4 ${ }^{+}$T-cell loss, we normalized $\mathrm{CD} 4^{+} \mathrm{T}$-cell levels relative to the last prechallenge value measured for each animal (Figure 5b). Using the normalized index, relative $\mathrm{CD} 4^{+} \mathrm{T}$-cell levels were significantly greater in each immunized group than the sham group 4 weeks post-challenge, as well as at additional time points, with the exception of the DNA AE rAd group which had more 

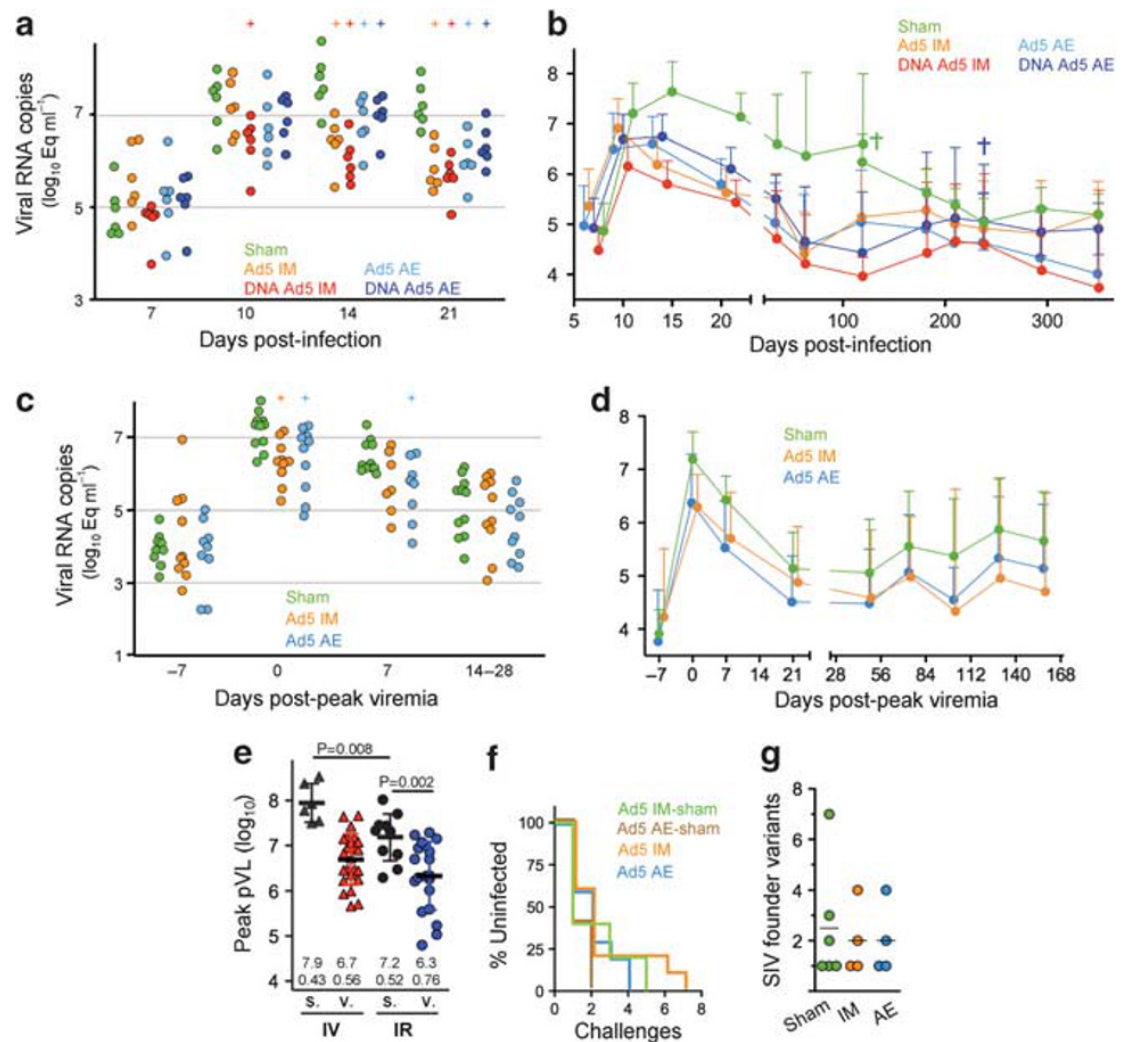

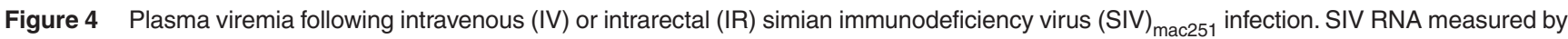
reverse transcription PCR is shown following IV infection by animal (a) and group averages (b); bars indicate 1s.d. above the mean; (+) indicates significant difference from the sham group $(P<0.05, t$-test). Mean plasma viral load $(\mathrm{pVL})$ is plotted with and without data for any animals that died (colored cross) before the subsequent time point. (c) pVL following IR SIV infection with peak viremia for each animal synchronized at week 0 . Aerosol (AE) and intramuscular (IM) sham groups were combined into a single group for comparison purposes. (d) Group mean pVL following IR infection is plotted as in b using animals' peak-synchronized viremia. (e) Peak pVL values are plotted for each animal from vaccinated (v.) and sham (s.) groups following IV and IR infection. Bars and values indicate the mean and $1 \mathrm{~s}$.d.; $t$-test $P$-values are shown (top). (f) Rate of IR SIV acquisition was determined by the first weekly plasma collection with detectable SIV RNA. (g) Transmitted SIV variants following IR challenge is plotted for a subset of animals. Founder population size was estimated by env sequence homology ( $n=10-20$ per animal) from plasma at peak viremia.

within-group variation. After the first month of infection, both AE groups recovered substantially, reaching approximately twice pre-challenge levels by week 17 , while sham animals remained below $25 \%$ of pre-challenge levels.

Jejunal CD4 ${ }^{+}$T-cell loss measured in biopsies from two animals per group (IV study) was rapid in the sham group, dropping to an average $7.3 \%$ at week 4 (Figure $5 \mathrm{c}$; pre-challenge average was $27 \%$ ). All immunized animals better maintained jejunal $\mathrm{CD} 4^{+} \mathrm{T}$ cells than sham animals 2 months post-challenge, although only the Ad5 $\operatorname{IM}(P=0.05)$ and DNA Ad5 $\operatorname{IM}(P=0.06)$ groups trended toward statistically significant preservation. Combined, these data suggest that IM and AE delivery offer similar protection against both lung and small intestine mucosal $\mathrm{CD} 4{ }^{+}$T-cell depletion following IV SIV challenge, with the possible exception of IM rAd5, which may enhance jejunal preservation during acute infection.

In the IR challenge setting, rAd5 immunization was less effective at mitigating mucosal CD4 ${ }^{+}$T-cell loss, which itself was more modest than that following IV challenge. Only the IM group had elevated BAL CD4 ${ }^{+} \mathrm{T}$ cells relative to the sham group 1 month after infection (Figure 5d). However, compared with pre-challenge values, neither immunized group preserved
$\mathrm{CD} 4^{+} \mathrm{T}$ cells better than the corresponding sham group early after infection, with all groups averaging 30-60\% reductions in $\mathrm{CD} 4^{+} \mathrm{T}$ cells (Figure 5e). The IR challenge sham animals had better recovery at later time points compared with sham animals following IV challenge (Figure 5b). Consequently, although the AE group achieved a 1.5-fold average increase by 9 weeks post-infection relative to pre-challenge levels, this was not significantly different from the sham AE group. Unlike the IV challenge, rAd 5 by either route failed to protect against $\mathrm{CD} 4^{+}$ $\mathrm{T}$-cell loss in the BAL, although the pathogenesis was considerably milder following IR challenge.

Surprisingly, in sharp contrast to IV administration, massive $\mathrm{CD} 4^{+}$T-cell depletion in the jejunum was not apparent following IR challenge (Figure 5f). The proportion of $\mathrm{CD}^{+} \mathrm{T}$ cells in the sham group ranged from $6 \%$ to $28 \%$ (mean 16.9) 5 weeks after infection and 6-22\% (mean 12.2) at week 10, suggesting a milder insult to this compartment (average CD4 frequency in uninfected historic controls is $30 \%$ ). Moreover, there was no effect of rAd5 immunization on jejunal $\mathrm{CD} 4^{+} \mathrm{T}$-cell representation. Thus, lowdose IR challenge does not deplete jejunal $\mathrm{CD} 4^{+} \mathrm{T}$ cells to the same extent as IV challenge and immunization offered no protection against the small deficit that was observed. 

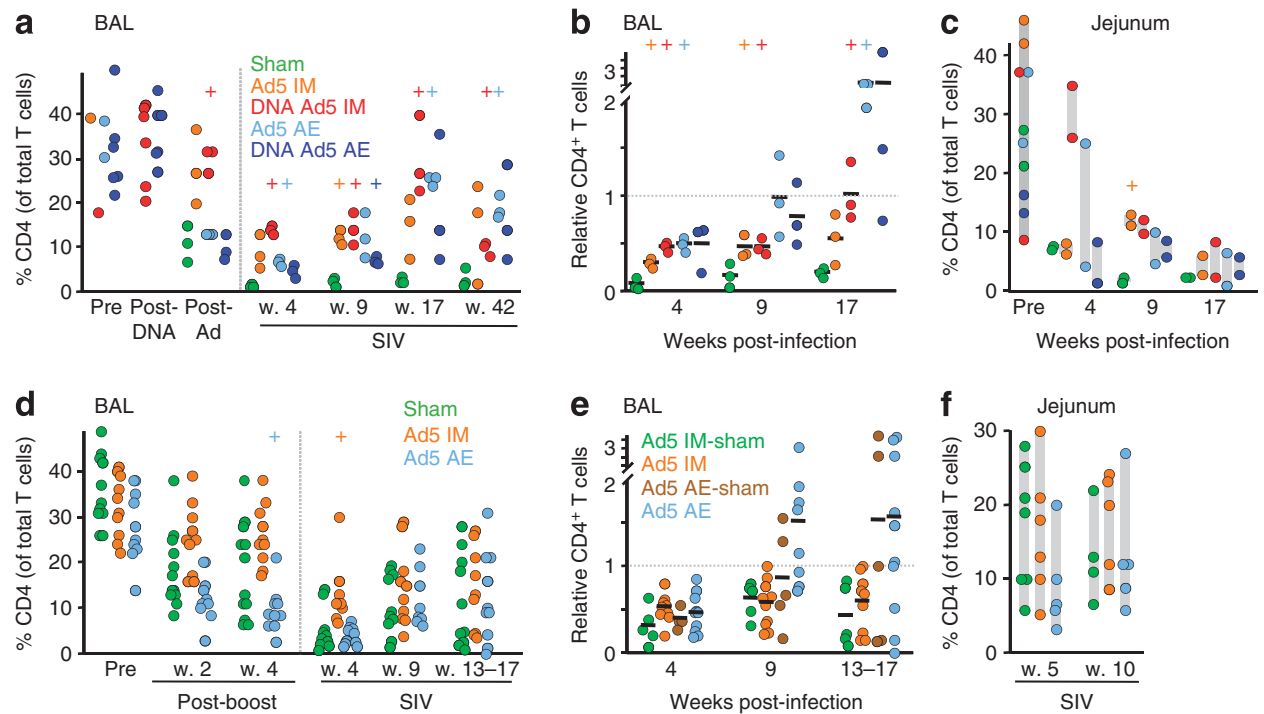

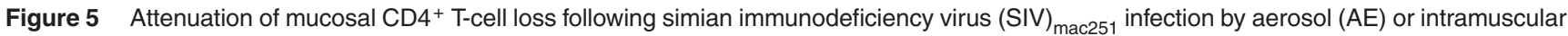
(IM) recombinant adenovirus serotype 5 (rAd5). (a) Percent CD4 ${ }^{+}$cells among bronchoalveolar lavage (BAL) $\mathrm{T}$ cells for three animals randomly selected from each group is plotted at the indicated time following immunization and intravenous (IV) infection. + Indicates $P<0.05$ ( $t$-test) compared with the sham group. (b) CD4 ${ }^{+}$T-cell percentage in the BAL following IV infection, shown in a, was divided by pre-infection (5 weeks post-rAd5 boost) for each animal at the indicated weeks post-infection as a relative indicator of CD4 ${ }^{+}$T-cell loss. Black bars indicate the group mean. (c) Jejunal CD4 ${ }^{+}$ T-cell representation following IV challenge was measured as in a for two additional animals in each group. Pre-challenge values for all 10 animals are shown at left. (d) BAL CD4+ T-cell percentage as in a for the intrarectal (IR) study (all animals shown). Sham groups were combined into a single group for comparison purposes. (e) CD4 ${ }^{+}$T-cell loss in the BAL was calculated as in $\mathbf{b}$ following IR challenge. (f) Jejunal CD4 ${ }^{+}$T-cell representation following IR infection as in $\mathbf{c}$ for $n=5-6$ animals from the IM and AE groups, and $n=4-6$ animals from the combined sham groups.

\section{Post-challenge anamnestic cellular responses}

To determine whether immunization augmented cellular immune responses mounted against the SIV challenge, we measured the frequency of SIV-specific T cells in the blood, BAL, and jejunum post-challenge. Aerosolized rAd5 primed for an anamnestic $\mathrm{CD} 8{ }^{+} \mathrm{T}$-cell response in peripheral blood mononuclears cell that exceeded the primary response in the sham group following both IV and IR infection (Figure 6a and b). However, these responses were weaker than those generated by systemic vaccination and benefited from a systemic DNA prime. DNA priming also altered the composition of the anamnestic $\mathrm{CD} 4{ }^{+}$ T-cell peripheral blood response, shifting the dominant specificity from Gag to Env 5 weeks post-infection (Supplementary Figure S3A online). In the BAL, anamnestic $\mathrm{CD} 8^{+}$and $\mathrm{CD} 4^{+}$ $\mathrm{T}$-cell responses were not significantly different between the AE and IM rAd5 groups following IV challenge (Figure 6c). By contrast, after mucosal challenge, AE rAd5 immunization primed for a significantly larger $\mathrm{CD} 4{ }^{+} \mathrm{T}$-cell recall response in the BAL than the IM route (Figure $6 \mathbf{d}$ ), suggesting that mucosal immunization primes for a greater mucosal anamnestic T-cell response to infection when the challenge is delivered mucosally. This effect is specific for the $\mathrm{CD} 4^{+}$subset and the site of immunization, as AE delivery did not enhance the anamnestic jejunal T-cell response, regardless of the challenge route (Supplementary Figure S3B-D online, and week 4 data not shown). Notably, peak $\mathrm{BAL} \mathrm{CD}^{+}$and $\mathrm{CD} 8{ }^{+} \mathrm{T}$-cell responses were generally greater for all vaccinated groups to the IR challenge than the IV challenge (Figure 6e). Thus, the anamnestic T-cell response elicited in the BAL during acute IR SIV infection exceeds that generated by high-dose systemic challenge.

\section{Immune correlates of protection}

To determine whether cellular or humoral immune responses correlated with clinical outcomes of infection, we performed linear regression and nonparametric Spearman's rank analyses between the magnitude of measured immunological parameters and plasma viremia and mucosal CD4 ${ }^{+}$T-cell depletion (Figure 7 and Table 1). Reduced peak viremia was strongly associated with the magnitude of SIV-specific CD8 ${ }^{+}$T-cell responses in the BAL in both the IV and IR challenge groups at multiple time points post-infection (Figure 7a and b). Furthermore, these responses inversely correlated with plasma viremia set point following IR SIV challenge (Table 1), suggesting that the mucosal CD8 ${ }^{+} \mathrm{T}$-cell response reduced viremia. Similarly, blood $\mathrm{CD}^{+} \mathrm{T}$-cell responses consistently correlated with reduced peak viremia, including vaccination-elicited responses before SIV challenge (Table 1). Preservation of mucosal (BAL) $\mathrm{CD}^{+}{ }^{+} \mathrm{T}$ cells following SIV challenge was also associated with the magnitude of the mucosal T-cell response, with a significant inverse correlation between mucosal CD4 ${ }^{+} \mathrm{T}$-cell depletion and the magnitude of both preand post-challenge $\mathrm{T}$-cell responses in the BAL: in IV-challenged animals both $\mathrm{CD}^{+}{ }^{+}$and $\mathrm{CD} 8{ }^{+} \mathrm{T}$-cell subsets correlated, whereas in IR-challenged animals only CD $4^{+} \mathrm{T}$-cell responses correlated. Together, these data indicate that T-cell responses likely aided in controlling virus replication and pathogenesis.

We performed similar analyses on pre-challenge antibody responses. In the IV-challenge setting, the only correlates of peak plasma viremia were nasal Env- and GagPol-specific IgG responses induced by DNA immunization (Figure 7c and Table 1). By contrast, preservation of $\mathrm{CD} 4{ }^{+} \mathrm{T}$ cells in the BAL was associated with Env-specific IgA responses at multiple sites 
a
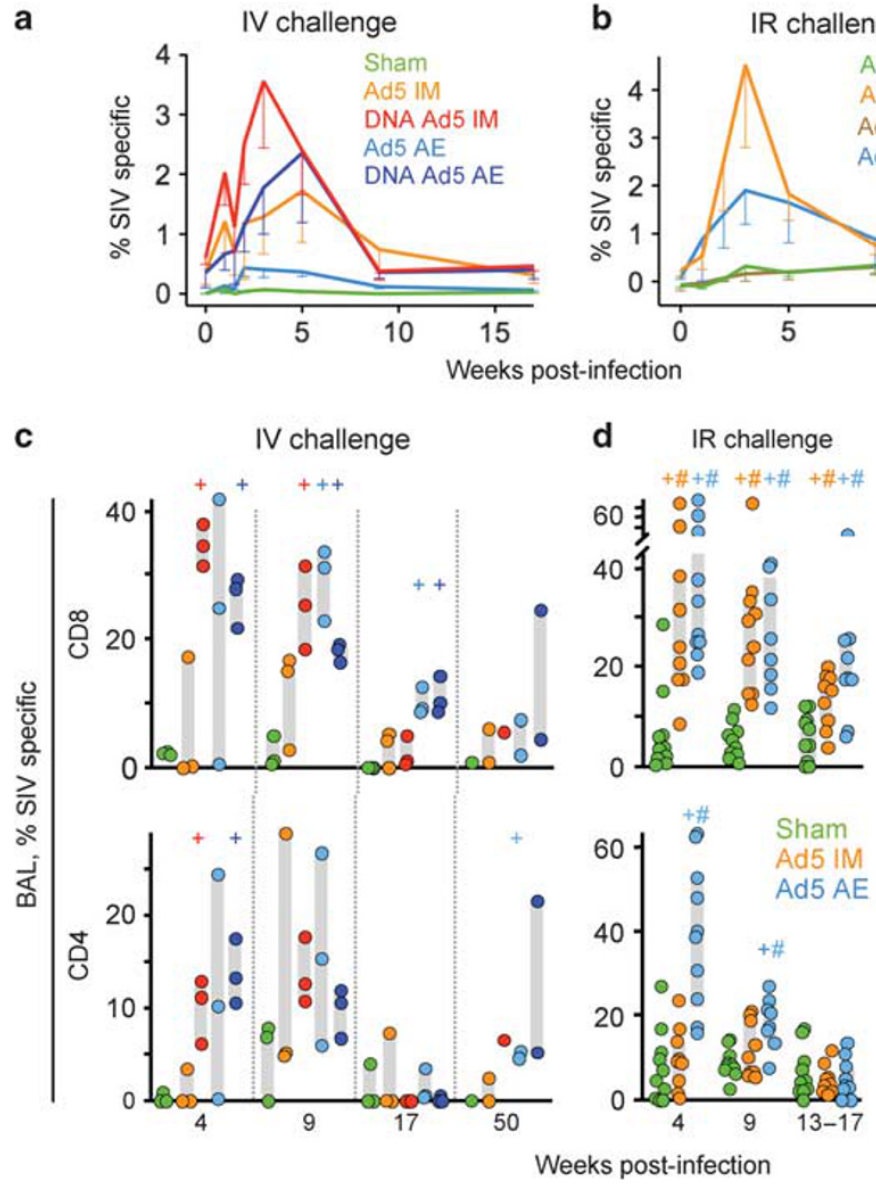

b IR challenge

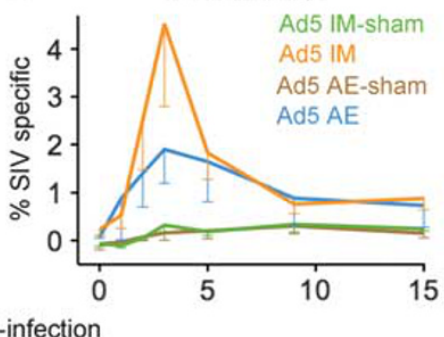

e Week 4
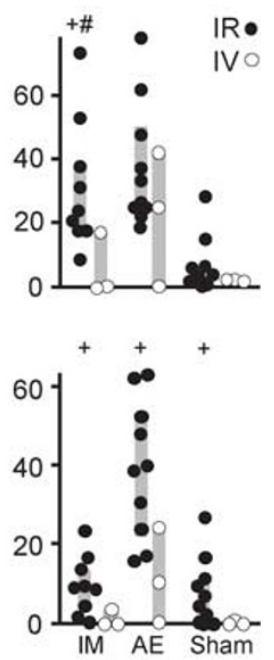

Figure 6 Simian immunodeficiency virus (SIV)-specific cellular responses following intravenous (IV) and intrarectal (IR) SIV challenge. (a) The anamnestic SIV-specific peripheral blood CD8 ${ }^{+}$T-cell response is plotted as the mean response for each vaccination group at the indicated time following IV and IR (b) infection. Responses were measured by intracellular cytokine staining as in Figure 3a, with Gag-and Env-reactive cells shown in a, and Gag, Pol, and Env shown in b. Error bars indicate 1 s.e. below the mean. (c) SIV-specific CD8 ${ }^{+}$and CD4 ${ }^{+} \mathrm{T}_{\text {cells }}$ in the bronchoalveolar lavage (BAL) following IV ( $n=3$ per group) and IR (d) challenge is plotted at the indicated weeks post-infection. The sham groups from the IR challenge study were combined into a single group for analysis. Gray bars indicate the interquartile range for each group. + ( $t$-test) or \# (Wilcoxon) indicates $P<0.05$ compared with the sham ( $\mathrm{AE}+\mathrm{IM}$ ) group. (e) Comparison of BAL anamnestic responses following IV or IR challenge for CD8 ${ }^{+}$and $\mathrm{CD}^{+}{ }^{+} \mathrm{T}$ cells at week 4 post-infection. AE, aerosol; IM, intramuscular.

following the rAd5 boost, including serum, BAL, and nasal secretions (Table 1). For the IR study, anti-Env IgG responses in plasma correlated with preservation of jejunal $\mathrm{CD} 4^{+} \mathrm{T}$ cells, but not with any viremia measurements (Figure 7d and Table 1). Although there was no protective efficacy against IR SIV infection among vaccinated animals, analysis of their responses as a group may identify potential correlates for other clinical end points, such as acquisition rate. ${ }^{26}$ However, no association was observed between plasma IgG (anti-Env or anti-Gag) responses and SIV infection rate or viremia among animals with significant responses to the vaccine (data not shown). Pre-challenge SIV-specific T-cell responses also failed to associate with the rate of SIV infection. Thus, humoral responses correlated with maintenance of mucosal $\mathrm{CD} 4^{+} \mathrm{T}$ cells in both challenge studies and with peak viremia in the IV study.

\section{DISCUSSION}

Aerosolized adenovirus afforded the same protection against viremia and pathogenicity in an IV SIV mac251 $_{1}$ challenge model as
IM delivery, despite the lack of peripheral T-cell responses and neutralizing antibodies at the time of challenge. This included reduced viremia throughout acute infection and attenuated mucosal CD4 ${ }^{+}$T-cell depletion. SIV inoculated rectally was also controlled to a similar extent by these disparate rAd5 delivery routes, although vaccination did not impact disease indicators as dramatically as in the IV model. Specifically, rAd5 immunization lowered peak viremia, but not other acute time points, while mucosal $\mathrm{CD} 4^{+} \mathrm{T}$-cell depletion, which was much milder in this model, was not significantly ameliorated by immunization. To our knowledge, these data represent the first test of mucosal replication-deficient $\mathrm{rAd} 5$ vaccination against mucosal SIV challenge and demonstrate that $\mathrm{AE}$ immunization provides at least comparable protection to IM delivery.

The similar IV challenge outcome for the AE and IM groups was not anticipated based on their respective pre-challenge immunogenicity profiles. No T-cell responses were detected in blood at the time of challenge in the AE group; thus, circulating virus-specific $\mathrm{T}$ cells did not predict virus control in 

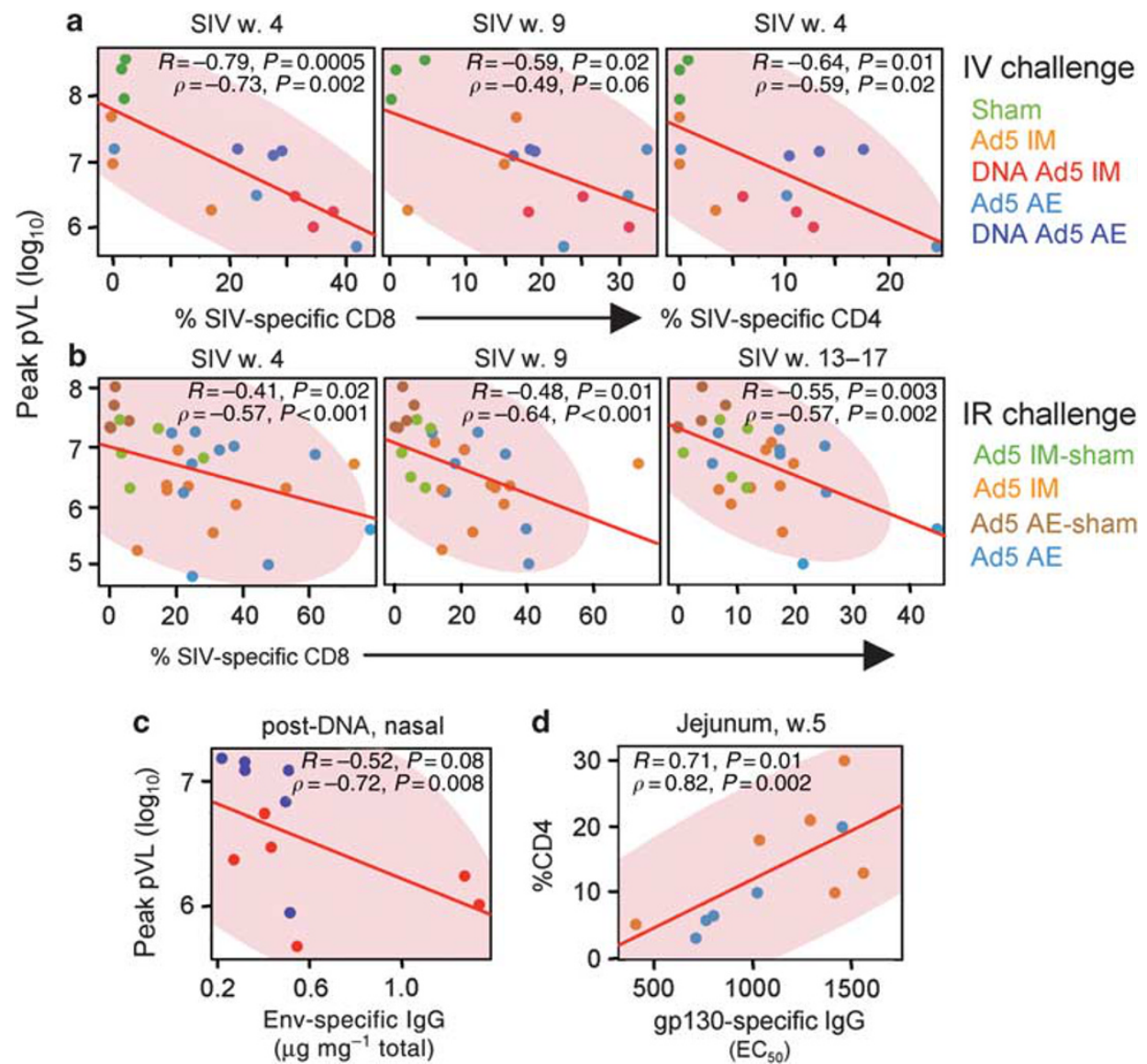

Figure 7 Post-challenge mucosal immune correlates of viremia control and mucosal CD4+ T-cell preservation. Scatterplots depicting linear regression (red line; $R$ ) and nonparametric Spearman's rank correlation $(\rho)$ analysis with corresponding $P$-values. Shaded area depicts the $95 \%$ bivariate normal density ellipse for the nonparametric analysis. (a) Correlation between simian immunodeficiency virus (SIV)-specific bronchoalveolar lavage (BAL) T-cell responses (as in Figure 3c) and peak plasma viremia following intravenous (IV) and intrarectal (IR) (b) challenge. The CD8 ${ }^{+}$and $\mathrm{CD}^{+}{ }^{+}$T-cell response magnitude at the indicated weeks post-infection is plotted against viremia for each animal. (c) Antibody immune correlates of plasma viremia control following IV challenge. Immunoglobin (Ig) responses after DNA immunization for nasal secretions are plotted against peak viremia. (d) Pre-challenge antibody correlates of SIV control following IR challenge for immunized animals with positive responses to the vaccine.

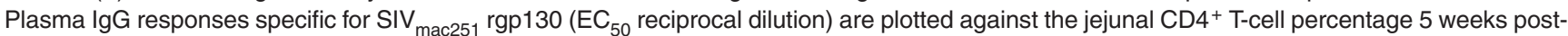
infection. AE, aerosol; w., week.

these animals. A similar disconnect was previously described for animals immunized with replication-competent Ad5 host range mutant vaccination, in which oral/oral delivery was much less immunogenic than combination oral/intranasal delivery, although both regimens controlled IR SIV mac251 $_{1}$ challenge to the same extent. ${ }^{27}$ Thus, mucosally derived T cells from tissues targeted by mucosal vaccines and associated draining lymph nodes are able to contribute to systemic virus control. This suggests that lymphocytes residing or primed in classical "effector" sites may be an important source of immunity, and commonly used assays measuring cellular immunogenicity in the blood may not correlate with vaccine efficacy against systemic or mucosal challenges.

It was also unexpected that the $\mathrm{AE}$ immunization did not provide greater protection against mucosal challenge than IM delivery. We hypothesized that T- and B-cell responses mounted in the airways would enhance rectal immunity to a greater extent than systemic delivery based on studies demonstrating a benefit to airway delivery: nasal vaccination was superior to
IM vaccination in protecting against IR SIV challenge with a DNA/recombinant-modified vaccinia virus Ankara platform; ${ }^{28}$ and lower respiratory tract intratracheal delivery offered superior protection against IR SIV challenge than combined oral and nasal delivery in a replication-competent Ad5 vaccination model. ${ }^{2}$ However, we did not detect significant rectal humoral or jejunal cellular responses following rAd5 administration by either route; therefore, $\mathrm{rAd} 5$ is likely insufficient to induce robust mucosal responses at distal sites. This is supported by our observations of (i) significantly higher rectal IgA when $\mathrm{AE}$ rAd5 is primed by systemic DNA compared with an unprimed response (Figure 2d); and (ii) greater jejunal $\mathrm{CD} 8^{+} \mathrm{T}$-cell responses elicited by DNA-primed IM rAd5 than unprimed IM rAd5 (Supplementary Figure S1 online). The only evidence that $\mathrm{AE}$ vaccination impacted the mucosal challenge outcome was a significantly greater anamnestic $\mathrm{BAL} \mathrm{CD} 4^{+} \mathrm{T}$-cell response to SIV (Figure 6d).

Why peak viremia values differed with the challenge route is unclear, although our IR values are consistent with lower 
Table 1 Summary of immune correlates of SIV disease protection ${ }^{a}$

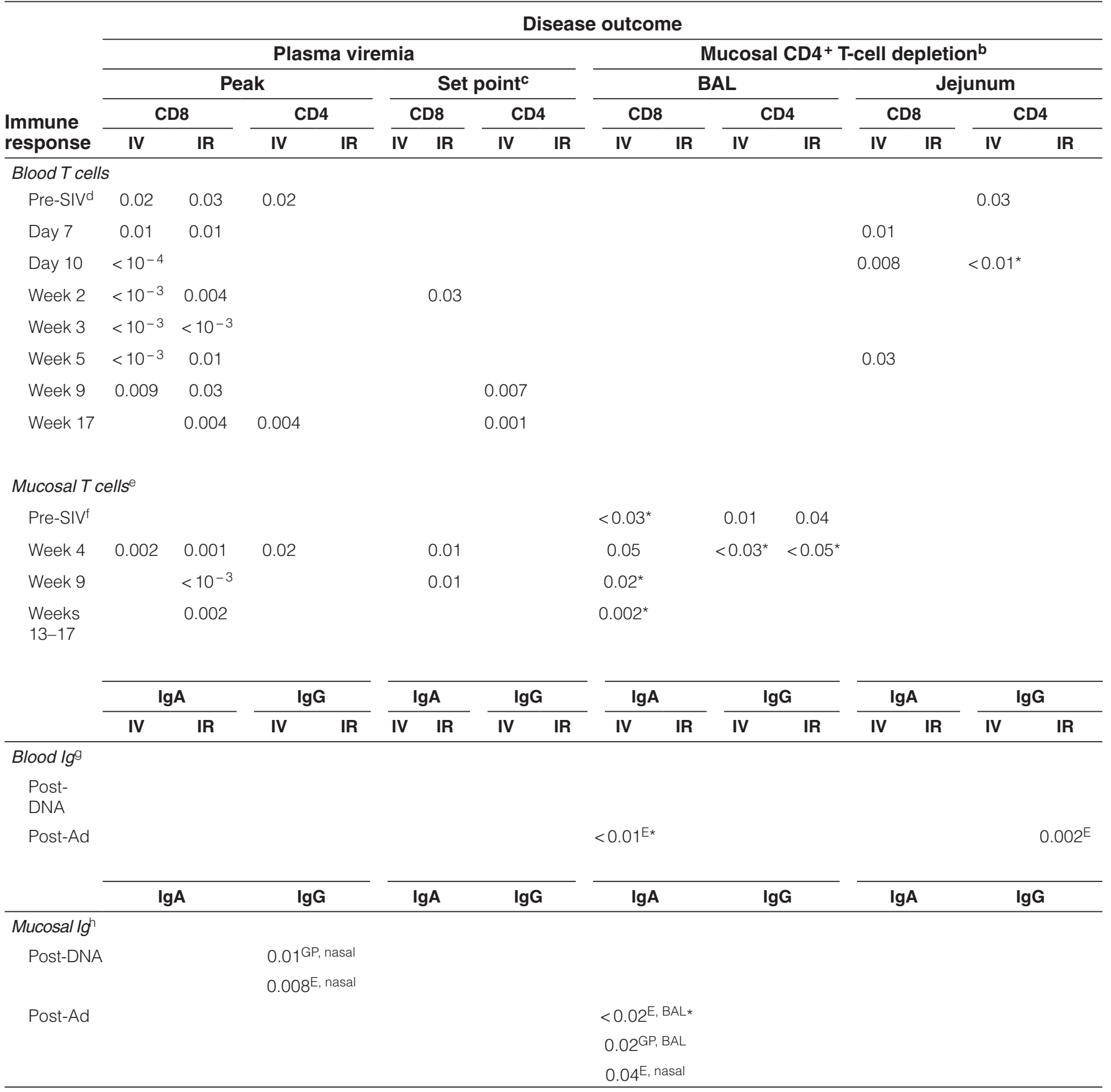

AE, aerosol; BAL, bronchoalveolar lavage; IM, intramuscular; IR, intrarectal; IV, intravenous; SIV, simian immunodeficiency virus.

aNonparametric Spearman's rank correlation P-values are listed for immune responses (at left) associated with a significant $(P<0.05)$ reduction in the indicated disease parameters (at top).

${ }^{b} B A L$ and jejunal CD4 ${ }^{+}$T-cell loss was measured as described in Figure $\mathbf{5}$ at 1, 2, and 3-4 months post-infection. Significant correlations at one of these time points are indicated by a single P-value; correlations at multiple time points are indicated by $\left(^{*}\right)$ with the largest $P$-value listed.

cPlasma viremia set point was calculated as the average copy number detected from month 6 (IV study) or month 4 (IR study) post-infection to the end of the study.

dPre-SIV correlation analyses do not include sham group animals and reflect responses at any time point prior to challenge.

eMucosal T-cell responses were measured in the BAL.

fSignificant pre-challenge correlations in the IR challenge study existed for AE immunized $(n=10)$ animals only.

glmmunoglobulin (Ig) specificity is indicated using superscript abbreviations as follows: Env (E), Gag (G), and GagPol (GP). IgA and IgG were measured for the IV study, while only IgG was measured for the IR study.

hMucosal Ig samples were not analyzed for the IR challenge study. The site of mucosal Ig is indicated. Rectal and vaginal samples were also analyzed, but did not yield significant associations. 
values observed by other repeated limiting-dose IR challenge studies. ${ }^{28,29}$ This may be due to slower initiation of infection following IR inoculation, requiring several days for a small number of viruses to cross the mucosal barrier and replicate to levels achieved within a single day following IV or high-dose mucosal transmission. Our data suggest that such IR inoculations result in a highly asynchronous acute infection process that may not lend itself to accurate peak viremia quantification by weekly samplings.

Preservation of $\mathrm{CD} 4^{+} \mathrm{T}$ cells in the small intestine, and to a lesser extent in the BAL, following the IR challenge in control and immunized animals was remarkable and unanticipated. Mucosal immunopathogenesis following IR SIV challenge has not been studied extensively, but several reports document severe $\mathrm{CD} 4{ }^{+} \mathrm{T}$-cell depletion in rectal mucosa within $2-3$ weeks of infection..$^{28,30,31}$ The reported effects on the small intestine are less consistent, ranging from no significant loss up to 2 weeks after infection to depletion commencing on day 7 or $21 .{ }^{31-33}$ This discrepancy may be related to the dose of the IR challenge virus, which may determine viral dissemination and $\mathrm{CD} 4^{+}$ T-cell cytopathicity in the upper gastrointestinal tract. Although the basis for this difference remains to be explored, our findings in the BAL were consistent with previously published findings from high-dose IR infection, demonstrating lung $\mathrm{CD} 4^{+} \mathrm{T}$-cell depletion by day $21,{ }^{31}$ suggesting that pathogenesis of rectal SIV challenge is less variable in the lung than in the gastrointestinal tract. Ultimately, mucosal challenge may favor a more rapid induction of the mucosal immune response, curbing viral replication and $\mathrm{CD}^{+}{ }^{+} \mathrm{T}$-cell depletion at mucosal sites.

The blood T-cell response correlates observed here are consistent with the well-documented contribution of both pre- and post-challenge responses to viremia control in IV and IR SIV challenge models. ${ }^{3,28,34-37}$ Importantly, we revealed an association between mucosal T-cell responses (primarily CD8) and viremia control as well as partial preservation of mucosal $\mathrm{CD} 4^{+}$ $\mathrm{T}$ cells. The demonstration that these mucosally derived $\mathrm{T}$ cells can limit viral replication and pathogenesis is consistent with evidence that effector memory $\mathrm{T}$ cells residing in mucosa protect against mucosal SIV replication. ${ }^{1}$

Pre-challenge antibody responses did not predict viral control to the extent observed for cellular responses. However, the association between pre-challenge respiratory tract IgA responses and retention of BAL CD4 $4^{+}$T cells suggests that local antibodies limited pathogenesis. While we were unable to identify cellular or humoral correlates of IR SIV acquisition rate, it will be important to include mucosal IgA responses in these analyses in future studies. Despite this being a fairly large study by nonhuman primate standards, it is a relatively small study for the purpose of searching for immune correlates; our findings should be considered a guide for identifying and refining correlates in future studies. Certainly, we cannot exclude the possibility of other correlates that did not rise to statistical significance in our study.

Together, these data show comparable efficacy between systemic and $\mathrm{AE}$ rAd5 vaccination against $\mathrm{SIV}_{\text {mac251 }}$-induced disease, regardless of the challenge route. Although we did not see any clear benefit of AE over IM immunization, comparing these regimens in the less stringent SIV $_{\text {smE660 }}$ mucosal challenge model may better discriminate between their protective capacities. We demonstrate that the addition of a systemic DNA prime enhanced the distal mucosal humoral responses elicited by AE rAd5. In addition, we identified discrepancies in the pathogenesis of IV and IR SIV infection in the macaque model, raising the question of which model accurately reproduces HIV disease. We conclude that the AE modality represents a useful immunization vehicle for vaccine vectors not only against respiratory pathogens such as influenza, measles, and tuberculosis, but that it may also complement vaccine strategies against HIV and other pathogens by inducing excellent cellular and humoral immunity.

\section{METHODS}

Immunizations and SIV challenge. Colony-bred Indian-origin rhesus macaques were immunized with three plasmid DNA priming immunizations 4 weeks apart in the IV study, consisting of $4 \mathrm{mg}$ each of

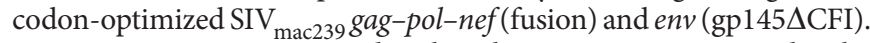
Immunogens were expressed within the vector pVR1012 under the control of cytomegalovirus immediate-early enhancer, promoter, and first intron. Delivery was IM in the anterior quadriceps by Biojector. ${ }^{38}$ Recombinant E1/E3/E4-deleted rAd5 constructs and virus stocks were generated as previously described. ${ }^{39-41}$ The active study arms $(n=6$, IV study; $n=10$ IR study) received $5 \times 10^{10}$ each of rAd5 expressing $\mathrm{GagPol}_{\text {SIV }}$ and $\mathrm{Env}_{\mathrm{SIV}}(\mathrm{gp} 140)$, while control groups $(n=6)$ received $10^{11}$ particles of empty rAd5. Immunizations were given twice, either 21 (IV) or 25 (IR) days apart and 26 weeks after the last DNA prime in the IV study. rAd5 was delivered either IM by needle and syringe in the right quadriceps or by AE using the e-Flow Nebulizer System (PARI Pharma, Munich, Germany). ${ }^{11}$

Animals were challenged with $\mathrm{SIV}_{\text {mac251 }} \mathrm{IV}$ at $100 \mathrm{MID}_{50}$, while the IR challenge dose was predetermined to result in $50 \%$ infection. Virus preparations of $1.0 \mathrm{ml}$ were inoculated via the saphenous vein or a lubricated feeding catheter inserted into the rectum. IR inoculations were repeated weekly for up to 8 weeks until viremia was detected in weekly plasma sampling.

Antibody measurements. Rectal secretions were sampled by a modified wick method using Weck-Cel Spears (Windsor Biomedical, Newton, $\mathrm{NH}$ ) as previously described. ${ }^{42}$ For the IV challenge study, pre-immune and pre-challenge serum and rectal sponge elutions were analyzed for humoral responses by enzyme-linked immunosorbent assay as previously described. ${ }^{11,43}$ The concentration of antigen-specific IgA or IgG was divided by the concentration of total IgA or IgG for each sample to obtain "specific activity." Samples were considered to contain significant antibody if the specific activity was greater than or equal to mean +3 s.d.s of negative controls and 3.4-fold above the pre-immune value. For the IR challenge study, SIVspecific humoral IgG responses at the time of challenge were evaluated by a standardized binding antibody multiplex array as previously described. ${ }^{44,45}$ Positive and negative monkey sera controls were used in each assay, and the midpoint titer $\left(\mathrm{EC}_{50}\right)$ of each sample was calculated using a four-parameter logic fit. Positive responses to the vaccine were assessed as threefold over pre-immune values and at least 50 mean fluorescence intensity (MFI).

Cellular immune responses and CD4 depletion. BAL, jejunal biopsies, lymph nodes, and peripheral blood were collected from animals following immunization and/or SIV challenge. Single-cell suspensions were stimulated with overlapping peptide pools of SIV Env, Gag, or Pol at $2.0 \mu \mathrm{g} \mathrm{ml}^{-1}$ for $16 \mathrm{~h}$ as previously described. ${ }^{46,47}$ Following stimulation, cells were labeled with cell surface markers (CD4-Alexa700APC, CD8-QDot655, CD45RA-TRPE, and CD95-APC) and ViViD (to discriminate live/dead cells). Intracellular cytokine staining on fixed and permeabilized (BD 
Cytofix/Cytoperm, Becton Dickenson, Franklin Lakes, NJ) samples was performed with interferon- $\gamma$-fluorescein isothiocyanate, tumor necrosis factor- $\alpha$-Cy7PE, interleukin-2-phycoerythrin, and CD3-Cy7APC. CD4 ${ }^{+}$ T-cell depletion in the BAL and jejunum was assessed as the percent of $\mathrm{CD} 4{ }^{+}$cells within the live, $\mathrm{CD} 3^{+}$lymphocytes population. For post-infection BAL analyses, $\mathrm{CD} 4{ }^{+} \mathrm{T}$-cell loss was also calculated as a ratio to prechallenge, post-immunization values.

Plasma viral RNA quantitation. For the IV challenge study, viral RNA was extracted from plasma samples as described previously. ${ }^{48}$ To quantify viral replication, a fluorescent resonance energy transfer probebased real-time reverse transcription PCR assay to detect SIV gag RNA was used as described. ${ }^{48,49}$ For the IR challenge study, viral RNA was extracted using the QIAmp Viral RNA Mini Kit (Qiagen, Germantown, $\mathrm{MD})$, reverse transcribed using SuperScript III (Invitrogen, Carlsbad, $\mathrm{CA}$ ), and treated with RNase H (Roche Applied Science, Indianapolis, IN) to remove remaining RNA. Real-time PCR detection of SIV gag complementary DNA was performed using TaqMan Gene Expression Master Mix and standard BHQ quencher technology for product detection. Viral load values obtained from the real-time reverse transcription PCR assays used for the IV and IR studies did not vary.

Single-genome amplification of $\operatorname{SIV}_{\operatorname{mac} 251}$ and sequencing. From each plasma specimen, 20,000 viral RNA copies were isolated and immediately subjected to complementary DNA synthesis with the gene-specific antisense primer SIVEnvR1 5' -TGTAATAAATCCCTTCCAGTCCCCC $\mathrm{C}-3^{\prime}$ as previously described. ${ }^{50}$ The entire env gene was sequenced from each animal at peak viremia using a limiting dilution PCR, so only one amplifiable molecule is present in each reaction. The number of unique variants was determined as described previously. ${ }^{50}$ All sequences were deposited in GenBank with accession numbers JN000939-JN001158.

SUPPLEMENTARY MATERIAL is linked to the online version of the paper at http://www.nature.com/mi

\section{ACKNOWLEDGMENTS}

We thank M. Louder and Dr J Mascola for performing SIV Env neutralization assays and Dr J Lifson of SAIC-Frederick and Dr T Denny of Duke University for plasma viral load measurements. We thank Dr B. Lafont of the Laboratory of Molecular Microbiology, National Institute of Allergy and Infectious Diseases, for major histocompatibility complex haplotype sequencing. We also thank JP Todd and A Ault for assisting with animal immunizations, challenges, and sampling scheduling, as well as T Jenkins and A Dodson of Bioqual for animal care and handling. Adenovirus viral stocks were kindly provided by J Gall of GenVec. We thank Vicki C Ashley and R Glenn Overman for technical assistance with the antibody measurements and all members of the ImmunoTechnology Section for insightful discussions. This work was supported in part by the National Institutes of Health grant AI058896 (to PAK) and the Louisiana Vaccine Center and the South Louisiana Institute for Infectious Disease Research sponsored by the Louisiana Board of Regents, by the Intramural Research Program of the National Institute of Allergy and Infectious Diseases, National Institutes of Health, and by the National Cancer Institute, National Institutes of Health under contract HHSN266200400088C.

\section{AUTHOR CONTRIBUTIONS}

DLB performed experimental design, most of the data analysis and interpretation, and writing of the manuscript. KS performed in vitro T-cell functional tests and analyses. RLW, PAK, and GDT performed in vitro antibody tests and analyses. BK performed viral sequencing experiments. RVL performed viral quantification experiments. DLB, SR, and MR formulated the original experimental idea and study design. All authors contributed to the writing of the manuscript.

\section{DISCLOSURE}

The authors declared no conflict of interest.

\section{REFERENCES}

1. Hansen, S.G. et al. Effector memory T cell responses are associated with protection of rhesus monkeys from mucosal simian immunodeficiency virus challenge. Nat. Med. 15, 293-299 (2009).

2. Pinczewski, J. et al. Enhanced immunity and protective efficacy against SIVmac251 intrarectal challenge following ad-SIV priming by multiple mucosal routes and gp120 boosting in MPL-SE. Viral. Immunol. 18, 236-243 (2005).

3. Zhao, J. et al. Improved protection of rhesus macaques against intrarectal simian immunodeficiency virus SIV(mac251) challenge by a replicationcompetent Ad5hr-SIVenv/rev and Ad5hr-SIVgag recombinant priming/ gp120 boosting regimen. J. Virol. 77, 8354-8365 (2003).

4. Crotty, S. et al. Protection against simian immunodeficiency virus vaginal challenge by using Sabin poliovirus vectors. J. Virol. 75, 7435-7452 (2001).

5. Enose, Y. et al. Protection by intranasal immunization of a nef-deleted, nonpathogenic SHIV against intravaginal challenge with a heterologous pathogenic SHIV. Virology 298, 306-316 (2002).

6. Buchbinder, S.P. et al. Efficacy assessment of a cell-mediated immunity HIV-1 vaccine (the Step Study): a double-blind, randomised, placebo-controlled, test-of-concept trial. Lancet 372, 1881-1893 (2008).

7. Liu, J. et al. Immune control of an SIV challenge by a T-cell-based vaccine in rhesus monkeys. Nature 457, 87-91 (2009).

8. Santra, S. et al. Heterologous prime/boost immunizations of rhesus monkeys using chimpanzee adenovirus vectors. Vaccine $\mathbf{2 7}, 5837-5845$ (2009).

9. Patterson, L.J. et al. Protection against mucosal simian immunodeficiency virus SIV(mac251) challenge by using replicating adenovirus-SIV multigene vaccine priming and subunit boosting. J. Virol. 78, 2212-2221 (2004).

10. Schulte, R. et al. Mucosal prior to systemic application of recombinant adenovirus boosting is more immunogenic than systemic application twice but confers similar protection against SIV-challenge in DNA vaccine-primed macaques. Virology 383, 300-309 (2009).

11. Song, K. et al. Genetic immunization in the lung induces potent local and systemic immune responses. Proc. Natl. Acad. Sci. USA 107, 22213-22218 (2010)

12. Balmelli, C., Demotz, S., Acha-Orbea, H., De Grandi, P. \& NardelliHaefliger, D. Trachea, lung, and tracheobronchial lymph nodes are the major sites where antigen-presenting cells are detected after nasal vaccination of mice with human papillomavirus type 16 virus-like particles. J. Virol. 76, 12596-12602 (2002).

13. Nardelli-Haefliger, D. et al. Immune responses induced by lower airway mucosal immunisation with a human papillomavirus type 16 virus-like particle vaccine. Vaccine 23, 3634-3641 (2005).

14. Cutts, F.T., Clements, C.J. \& Bennett, J.V. Alternative routes of measles immunization: a review. Biologicals 25, 323-338 (1997).

15. Kannanganat, S. et al. Preexisting vaccinia virus immunity decreases SIV-specific cellular immunity but does not diminish humoral immunity and efficacy of a DNA/MVA vaccine. J. Immunol. 185, 7262-7273 (2010).

16. Letvin, N.L. et al. Immune and genetic correlates of vaccine protection against mucosal infection by SIV in monkeys. Sci. Transl. Med. 3, 81ra36 (2011).

17. Holmgren, J. \& Czerkinsky, C. Mucosal immunity and vaccines. Nat. Med. 11, S45-S53 (2005).

18. Darrah, P.A. et al. Multifunctional TH1 cells define a correlate of vaccinemediated protection against Leishmania major. Nat. Med. 13, 843-850 (2007).

19. Harari, A., Petitpierre, S., Vallelian, F. \& Pantaleo, G. Skewed representation of functionally distinct populations of virus-specific CD4 T cells in HIV-1-infected subjects with progressive disease: changes after antiretroviral therapy. Blood 103, 966-972 (2004).

20. Letvin, N.L. et al. Preserved CD4+ central memory T cells and survival in vaccinated SIV-challenged monkeys. Science 312, 1530-1533 (2006).

21. Loffredo, J.T. et al. Mamu-B*08-positive macaques control simian immunodeficiency virus replication. J. Virol. 81, 8827-8832 (2007).

22. Brenchley, J.M. et al. CD4+ T cell depletion during all stages of HIV disease occurs predominantly in the gastrointestinal tract. J. Exp. Med. 200, 749-759 (2004). 
23. Mattapallil, J.J., Douek, D.C., Hill, B., Nishimura, Y., Martin, M. \& Roederer, M. Massive infection and loss of memory CD4+ T cells in multiple tissues during acute SIV infection. Nature 434, 1093-1097 (2005).

24. Veazey, R.S. et al. Identifying the target cell in primary simian immunodeficiency virus (SIV) infection: highly activated memory CD4(+) T cells are rapidly eliminated in early SIV infection in vivo. J. Virol. 74, 57-64 (2000).

25. Zeng, X., Moore, T.A., Newstead, M.W., Deng, J.C., Lukacs, N.W. \& Standiford, T.J. IP-10 mediates selective mononuclear cell accumulation and activation in response to intrapulmonary transgenic expression and during adenovirus-induced pulmonary inflammation. J. Interferon. Cytokine Res. 25, 103-112 (2005).

26. Qin, L., Gillbert, P.B., Corey, L., McElrath, M.J. \& Self, S.G. A framework for assessing immunological correlates of protection in vaccine trials. J. Infect. Dis. 196, 1304-1312 (2007).

27. Zhou, Q. et al. Comparative evaluation of oral and intranasal priming with replication-competent adenovirus 5 host range mutant (Ad5hr)-simian immunodeficiency virus (SIV) recombinant vaccines on immunogenicity and protective efficacy against SIV(mac251). Vaccine 25, 8021-8035 (2007).

28. Manrique, M. et al. Nasal DNA-MVA SIV vaccination provides more significant protection from progression to AIDS than a similar intramuscular vaccination. Mucosal Immunol. 2, 536-550 (2009).

29. Wilson, N.A. et al. Vaccine-induced cellular immune responses reduce plasma viral concentrations after repeated low-dose challenge with pathogenic simian immunodeficiency virus SIVmac239. J. Virol. 80, 5875-5885 (2006)

30. Vaccari, M. et al. CD4+ T-cell loss and delayed expression of modulators of immune responses at mucosal sites of vaccinated macaques following SIV(mac251) infection. Mucosal Immunol. 1, 497-507 (2008)

31. Vajdy, M. et al. Early immunologic events in mucosal and systemic lymphoid tissues after intrarectal inoculation with simian immunodeficiency virus. J. Infect. Dis. 184, 1007-1014 (2001).

32. Couedel-Courteille, A. et al. Delayed viral replication and CD4(+) T cell depletion in the rectosigmoid mucosa of macaques during primary rectal SIV infection. Virology 316, 290-301 (2003).

33. Kewenig, S. et al. Rapid mucosal CD4(+) T-cell depletion and enteropathy in simian immunodeficiency virus-infected rhesus macaques. Gastroenterology 116, 1115-1123 (1999).

34. Leno, M. et al. CD8+ lymphocyte antiviral activity in monkeys immunized with SIV recombinant poxvirus vaccines: potential role in vaccine efficacy. AIDS Res. Hum. Retroviruses 15, 461-470 (1999).

35. Hel, Z. et al. Potentiation of simian immunodeficiency virus (SIV)specific CD4(+) and CD8(+) T cell responses by a DNA-SIV and NYVAC-SIV prime/boost regimen. J. Immunol. 167, 7180-7191 (2001).
36. Seth, A. et al. Immunization with a modified vaccinia virus expressing simian immunodeficiency virus (SIV) Gag-Pol primes for an anamnestic Gag-specific cytotoxic T-lymphocyte response and is associated with reduction of viremia after SIV challenge. J. Virol. 74, 2502-2509 (2000).

37. Barouch, D.H. et al. Reduction of simian-human immunodeficiency virus 89.6P viremia in rhesus monkeys by recombinant modified vaccinia virus Ankara vaccination. J. Virol. 75, 5151-5158 (2001)

38. Rao, S.S. et al. Comparative evaluation of three different intramuscular delivery methods for DNA immunization in a nonhuman primate animal model. Vaccine 24, 367-373 (2006).

39. Brough, D.E., Lizonova, A., Hsu, C., Kulesa, V.A. \& Kovesdi, I. A gene transfer vector-cell line system for complete functional complementation of adenovirus early regions E1 and E4. J. Virol. 70, 6497-6501 (1996).

40. Gall, J.G. et al. Rescue and production of vaccine and therapeutic adenovirus vectors expressing inhibitory transgenes. Mol. Biotechnol. 35, 263-273 (2007).

41. Rasmussen, H. et al. TNFerade Biologic: preclinical toxicology of a novel adenovector with a radiation-inducible promoter, carrying the human tumor necrosis factor alpha gene. Cancer Gene. Ther. 9, 951-957 (2002).

42. Kozlowski, P.A., Lynch, R.M., Patterson, R.R., Cu-Uvin, S., Flanigan, T.P. \& Neutra, M.R. Modified wick method using Weck-Cel sponges for collection of human rectal secretions and analysis of mucosal HIV antibody. J. Acquir. Immune. Defic. Syndr. 24, 297-309 (2000).

43. Letvin, N.L. et al. No evidence for consistent virus-specific immunity in simian immunodeficiency virus-exposed, uninfected rhesus monkeys. J. Virol. 81, 12368-12374 (2007).

44. Liu, J. et al. Low-dose mucosal simian immunodeficiency virus infection restricts early replication kinetics and transmitted virus variants in rhesus monkeys. J. Virol 84, 10406-10412 (2010).

45. Tomaras, G.D. et al. Initial B-cell responses to transmitted human immunodeficiency virus type 1: virion-binding immunoglobulin M (IgM) and IgG antibodies followed by plasma anti-gp41 antibodies with ineffective control of initial viremia. J. Virol 82, 12449-12463 (2008).

46. Betts, M.R. et al. Analysis of total human immunodeficiency virus (HIV)specific CD4(+) and CD8(+) T-cell responses: relationship to viral load in untreated HIV infection. J. Virol 75, 11983-11991 (2001).

47. Maecker, H.T. et al. Use of overlapping peptide mixtures as antigens for cytokine flow cytometry. J. Immunol. Methods 255, 27-40 (2001).

48. Cline, A.N., Bess, J.W., Piatak, M. Jr \& Lifson, J.D. Highly sensitive SIV plasma viral load assay: practical considerations, realistic performance expectations, and application to reverse engineering of vaccines for AIDS. J. Med. Primatol. 34, 303-312 (2005).

49. Lifson, J.D. et al. Role of CD8(+) lymphocytes in control of simian immunodeficiency virus infection and resistance to rechallenge after transient early antiretroviral treatment. J. Virol. 75, 10187-10199 (2001).

50. Keele, B.F. et al. Low-dose rectal inoculation of rhesus macaques by SIVsmE660 or SIVmac251 recapitulates human mucosal infection by HIV-1. J. Exp. Med. 206, 1117-1134 (2009). 